\title{
Reproductive life events and Alzheimer's disease in Italian women: a retrospective study
}

\author{
This article was published in the following Dove Press journal: \\ Neuropsychiatric Disease and Treatment \\ 20 November 2012 \\ Number of times this article has been viewed
}

\author{
Chiara Zucchella' \\ Elena Sinforiani' \\ Antonietta Citterio' \\ Valentina Giarracca ${ }^{2}$ \\ Giorgio Bono² \\ Marco Mauri ${ }^{2}$ \\ 'IRCCS National Neurological \\ Institute, C Mondino Foundation, \\ Pavia, Italy; ${ }^{2}$ Department of Neurology, \\ Ospedale di Circolo/Università \\ dell'Insubria, Varese, Italy
}

\begin{abstract}
The aim of our study was to understand the complex relationship between the major reproductive life events in women with Alzheimer's disease. In a retrospective, case-control study, 275 women with Alzheimer's disease (AD) and 276 control patients from a hospital population were enrolled from July 2007 to December 2010. The AD patients presented with later menopause $(P<0.01)$, lower occurrence of surgical menopause $(P<0.04)$, reduced hormonereplacement therapy intake $(P<0.0001)$, and a longer reproductive life span $(P<0.01)$, compared to controls. No significant differences were found with respect to age at puberty, number of pregnancies, previous abortions, or contraceptive therapy. A higher education level appeared to have a protective role against the risk of developing AD. In women, menopause and hormone-replacement therapy can differentially modulate the clinical manifestations of $\mathrm{AD}$, but these factors do not play a predictive role in its development.
\end{abstract}

Keywords: Alzheimer's disease, gender, menopause, hormone-replacement therapy, surgical menopause, education

\section{Introduction}

In recent years, considerable attention has been focused on the effect of gender on cognitive functions and the development of neurodegenerative disorders. It is well known that sex steroids, particularly estrogen, act on the central nervous system to influence neurotransmission and synaptic plasticity and to protect the central nervous system from a wide range of neurotoxic insults.

A higher prevalence of Alzheimer's disease (AD) in women than in men has been reported, at least in European studies. ${ }^{2}$ Various factors, including fewer comorbid diseases in women and the consequent increase in life expectancy ${ }^{3,4}$ have been suggested to explain this gender difference. In particular, the loss of the protective effects of estrogen in women after menopause, which has been demonstrated in vitro and in vivo, ${ }^{1-5}$ has been suggested as playing a role in the higher incidence of AD in women. However, studies on the effects of hormone-replacement therapy (HRT) on cognition (both as a prevention and as a therapy for AD) have produced controversial results, ${ }^{1-6}$ and some studies have even suggested an increased risk of dementia in women using HRT. ${ }^{5-7}$ Some researchers ${ }^{5,6,8-10}$ have hypothesized that there may be a "critical period" around menopause during which the administration of HRT may reduce the risk of developing AD later in life; this effect seems to be more significant in women under 49 years old. ${ }^{7,8}$ Other studies, however, have failed to detect the presence of this socalled window of opportunity. The duration of HRT seems to significantly affect the outcome, as positive effects were mainly seen in short-term studies ( $<4$ months). ${ }^{11}$ Nonetheless, HRT should not be initiated in older women. ${ }^{8,12,13}$
Correspondence: Elena Sinforiani

IRCCS C Mondino, via Mondino 2,

27100, Pavia, Italy

Tel +390382380290

Fax +390382380206

Email elena.sinforiani@mondino.it 
Undoubtedly, as compared to men, women experience increased cognitive vulnerability linked to endocrine fluctuations during their reproductive years. ${ }^{6}$ The menopausal transition is a particularly critical phase of cognitive vulnerability. Data from the literature suggest that natural menopause is not accompanied by substantial changes in cognitive abilities, although some studies suggest that women with later menopause have higher cognition. ${ }^{13,14}$ At any rate, when postmenopausal deficits occur, they usually affect cognitive speed and concentration; however, these impairments mainly reflect premenopausal, reduced cognitive efficiency rather than being a direct consequence of menopause. ${ }^{14}$ Moreover, in some women, a complex relationship exists among mood, memory, and hormones, which may negatively influence attention-mediated cognitive functions. ${ }^{15,16}$ Additionally, when surgical menopause occurs early, women may have a higher risk of developing cognitive deficits, particularly those affecting verbal episodic memory. ${ }^{13-17}$ However, the occurrence of an unfavorable biological milieu, which may be genetically determined, predisposes women to an increased risk of neurodegeneration. ${ }^{18}$

Taken together, these data suggest that the complex interactions among genetic predisposition, comorbidities, and biological and environmental factors that promote the development of $\mathrm{AD}$ are further influenced by the presence of hormonal fluctuations in women.

The scientific literature's main focus has been the influence of HRT and menopause on the occurrence of AD; the aim of this study was to further our understanding of the complex relationship between gender and dementia, considering all the major reproductive life events in women. In our analysis, besides reproductive life events, we also considered those factors known to be related to dementia (arterial hypertension, diabetes, cardiovascular diseases, and thyroid pathologies). ${ }^{19}$

\section{Materials and methods Subjects}

Female outpatients with AD (diagnosed in accordance with the NINCDS-ADRDA criteria ${ }^{20}$ ) who were referred between July 2007 and December 2010 to the Alzheimer Assessment Unit at the C Mondino Institute of Neurology Foundation in Pavia, Italy and to the Department of Neurology, Ospedale di Circolo, Varese, Italy were included in this retrospective study. The diagnostic evaluation included an objective neurological examination, a neuropsychological examination, and neuroimaging (computed tomography or magnetic resonance imaging). The control sample was composed of women aged 50 or more who were referred as outpatients to the same hospitals for noncognitive neurological complaints, including peripheral nervous system diseases, motor disturbances, anxiety, and headache. Patients with Parkinson's disease or cerebrovascular lesions (evidence of objective neurological signs and/or presence of neuroradiological correlates) were excluded. Therefore, the two groups of patients shared the same probability of admittance to the local health clinics for neurological problems. Additionally, the controls and the AD patients showed the same social and geographical distribution. The entire population, that is, both the $\mathrm{AD}$ patients and the controls, were menopausal.

\section{Measures}

All subjects underwent a structured interview for the collection of demographic and clinical characteristics. The following clinical parameters were considered: (1) family history of dementia; (2) age at puberty; (3) number of pregnancies; (4) previous abortions; (5) hormonal contraceptive therapy (at least 6 months); (6) age at menopause and type of menopause (physiological/surgical); (7) HRT (at least 6 months); (8) arterial hypertension; (9) diabetes; (10) cardiovascular diseases; (11) thyroid pathologies; (12) obesity; (13) reproductive system pathologies; (14) psychiatric diseases; (15) other pathologies (rheumatic, orthopedic, and endocrine [except thyroid] pathologies, among others); and (16) drug intake (classified as nonsteroidal anti-inflammatory drugs, anxiolytics, antidepressants, and neuroleptics, among others).

Conditions known to be related to dementia, such as arterial hypertension, diabetes, cardiovascular diseases, and thyroid pathologies (items 8-11, above), were considered separately from other pathological disorders that were clustered in item 15 . For items 8 through 16 , the onset before or after menopause was considered; for item 16, for AD patients, we considered only drug intake that occurred before disease onset. The difference between age at menopause and age at puberty was calculated, and termed "reproductive life span."

Patients' data were collected, with the caregivers' participation when needed. Where, despite the additional information of caregivers, information gaps remained, the patients were excluded from the study.

The Mini-Mental State Examination (MMSE) ${ }^{21}$ (Italian version $^{22}$ ) was administered to $\mathrm{AD}$ patients and controls to obtain a global cognitive evaluation. $\mathrm{AD}$ patients were also examined using the following measures: the Activities of Daily 
Living $^{23}$ scale to evaluate basic everyday activities (scores ranged from 0 to 6 - a higher score corresponded to a higher autonomy level); the Instrumental Activities of Daily Living ${ }^{24}$ scale to evaluate more-complex activities (scores ranged from 0 to $8-$ a higher score corresponded to a higher autonomy level); the Neuropsychiatric Inventory ${ }^{25}$ to evaluate the presence and severity of behavioral disturbances (scores ranged from 0 to 144 , with higher scores indicating greater behavioral disturbances); and the Clinical Dementia Rating ${ }^{26}$ to evaluate disease severity (scores ranged from 0 , which represented a lack of dementia, to 3 , which represented severe dementia).

\section{Statistical analysis}

The data describing the frequency and dispersion of the quantitative and qualitative variables, respectively, were submitted to univariate comparisons using the $\chi^{2}$ test for the discrete variables and analysis of variance (ANOVA) for the continuous variables. A multivariate comparison was performed with a regression model, including all the personnel and clinic variables related to the central investigation (reproductive life events) and those significantly different between the $\mathrm{AD}$ patients and the controls from the univariate analysis. The critical value for statistical significance was set at 0.05 . All analyses were conducted using SPSS version 17.0 for Microsoft Windows $^{\circledR}$ (SPSS Inc, Chicago, IL).

The study was carried out in accordance with the code of ethics of the World Medical Association (Declaration of Helsinki).

\section{Results}

Two hundred and seventy-five women with AD and 276 controls were enrolled in this study. Another 35 patients in both centers were excluded because anamnestic information was missing; these subjects did not differ in age or schooling. The ratio of $\mathrm{AD}$ patients to control patients was similar between the two clinics (Pavia clinic: 159:161; Varese clinic: 116:115); however, the age of the control patients at inclusion was lower for the Pavia clinic $(P<0.05)$.

The main clinical and demographic characteristics of the patients are reported in Table 1 . The controls were significantly more educated than the AD patients $(P<0.02)$, while more AD patients had a family history of dementia $(P<0.0001)$. As expected, the MMSE mean score was significantly lower for AD patients $(P<0.0001)$.

With respect to the major reproductive life events, the control subjects went through early menopause $(<45$ years) more frequently than the AD patients did $(P<0.01)$. Additionally, the controls had a higher occurrence of surgical menopause $(P<0.04)$, a greater HRT intake $(P<0.0001)$, and a shorter reproductive life span $(P<0.01)$. No significant differences were found with respect to age at puberty, number of pregnancies, prior abortions, or contraceptive therapy (Table 2).

We evaluated the presence of other pathological conditions (items 8 through 16) in both groups and their onset with respect to menopause (either pre- or postmenopausal); (Table 3). The control patients had a higher frequency of other pathologies (item 15) and a greater drug intake (regardless of the type of drug) as compared to the demented patients $(P<0.0001)$; however, no differences were detected with respect to the onset of these pathologies. Although no differences were found with respect to reproductive system pathologies between AD patients and controls,

Table I Clinical data and demographic characteristics of the AD and control patients (mean \pm standard deviation)

\begin{tabular}{|c|c|c|c|c|c|}
\hline & AD patients (275) & Controls (276) & Significance & df & $P$ \\
\hline Age (range) & $77.6 \pm 6.3(53-96)$ & $76.7 \pm 7.5(52-91)$ & $2.33^{\mathrm{a}}$ & I & 0.124 \\
\hline Schooling (years) & $6.1 \pm 2.9$ & $6.7 \pm 3.2$ & $5.32^{\mathrm{a}}$ & I & 0.02 \\
\hline Family history for dementia (yes/no) & $98 / 177$ & $61 / 215$ & $11.642^{b}$ & & 0.0001 \\
\hline Age at disease onset (years) & $74.7 \pm 6.2$ & - & & & \\
\hline Early-onset AD ( $\leq 65$ years), n (\%) & $18(6.5)$ & - & & & \\
\hline Late-onset AD (>65 years), n (\%) & $257(93.5)$ & - & & & \\
\hline Disease duration (range) & $2.9 \pm 1.6(1-10)$ & - & & & \\
\hline CDR & $1.6 \pm 0.6$ & - & & & \\
\hline MMSE (range) & $18.0 \pm 3.3(9-24)$ & $27.9 \pm 1.3(25-30)$ & $21.49^{a}$ & I & 0.0001 \\
\hline $\mathrm{ADL}$ & $5.1 \pm 1.1$ & - & & & \\
\hline IADL & $3.8 \pm 1.9$ & - & & & \\
\hline NPI & $4.6 \pm 3.3$ & - & & & \\
\hline
\end{tabular}

Notes: ${ }^{a} A N O V A ; ~ ' \chi^{2}$ test.

Abbreviations: AD, Alzheimer's disease; SD, standard deviation; CDR, Clinical Dementia Rating; MMSE, Mini-Mental State Examination; ADL, Activities of Daily Living; IADL, Instrumental Activities of Daily Living; NPI, Neuropsychiatric Inventory; df, degrees of freedom. 
Table 2 Major reproductive life events in the AD and control patients

\begin{tabular}{|c|c|c|c|c|c|}
\hline & AD patients (275) & Controls (276) & Significance & df & $P$ \\
\hline Age at puberty $\leq 10$ & 23 & 16 & $3.323^{b}$ & 2 & 0.190 \\
\hline $11-15$ & 227 & 243 & & & \\
\hline$>15$ & 25 & 17 & & & \\
\hline Number of pregnancies (mean $\pm S D$ ) & $1.8 \pm 1.3$ & $1.9 \pm 1.4$ & $0.75^{b}$ & $\mathrm{I}$ & 0.39 \\
\hline Abortion (yes/no) & $60 / 215$ & $64 / 212$ & $0.08^{\mathrm{b}}$ & I & 0.777 \\
\hline Contraceptive therapy (yes/no) & $8 / 267$ & $|5 / 26|$ & $1.611^{\mathrm{b}}$ & I & 0.204 \\
\hline Age at menopause $(<45)$ & $69 / 206$ & $95 / 181$ & $5.298^{\mathrm{b}}$ & $\mathrm{I}$ & 0.01 \\
\hline Menopause (physiological/surgical) & $246 / 29$ & $232 / 44$ & $3.037^{\mathrm{b}}$ & I & 0.04 \\
\hline HRT (yes/no) & $6 / 269$ & $32 / 244$ & $17.568^{\mathrm{b}}$ & $\mathrm{I}$ & 0.001 \\
\hline Reproductive life span (years, mean \pm SD) & $35.4 \pm 4.4$ & $34.5 \pm 5.0$ & $5.03^{b}$ & 1 & 0.01 \\
\hline
\end{tabular}

Notes: ${ }^{a} \mathrm{ANOVA}$; ${ }^{\mathrm{b}} \chi^{2}$ test.

Abbreviations: AD, Alzheimer's disease; SD, standard deviation; HRT, hormone replacement therapy; df, degrees of freedom; ANOVA, analysis of variance.

the latter showed a higher occurrence of reproductive system pathologies before menopause $(P<0.02)$.

We considered the effects of different categories of drugs separately. We selected two specific types of drugs for further analysis: nonsteroidal anti-inflammatory drugs and psychotropic drugs (including anxiolytics, antidepressants, and neuroleptics), as they were the most frequently used. The use of these drugs was significantly more frequent in the controls than in the $\mathrm{AD}$ patients $(P<0.0001)$, irrespective of the type of drug. When we considered the onset of $A D$ before or after menopause, only psychotropic drug intake before menopause was more frequent in the controls than in the AD patients $(P<0.0001)$. We did not separately consider the different types of pathologies, as the subgroups were too small to allow performing a statistical analysis.
Although we distinguished between early- and late-onset AD patients, only 18 (6.5\%) patients had an early onset of the disease (before 45 years); so statistical analysis to evaluate the impact of hormonal exposure on the risk of developing AD was not performed.

We then constructed a multivariate regression model, in which the dependent variable was the status of patient or control and the independent variables were the clinic attended, the level of schooling, the presence of family history for dementia, the age at menopause, physiological or surgical menopause, reproductive life span, HRT intake, the presence of other pathologies, drug intake, and the premenopausal occurrence of reproductive system pathologies, ie, conditions in which significant differences between the $\mathrm{AD}$ patients and controls have been reported. The schooling level $(P<0.05)$

Table 3 Presence of pathological conditions and their onset (pre-/postmenopause) in the AD and control patients $\left(\chi^{2}\right.$ test)

\begin{tabular}{|c|c|c|c|c|c|}
\hline & $\begin{array}{l}\text { AD patients } \\
(275)\end{array}$ & $\begin{array}{l}\text { Controls } \\
(276)\end{array}$ & Significance & df & $P$ \\
\hline Arterial hypertension (yes) & 161 & $|5|$ & 0.676 & I & 0.411 \\
\hline pre-/postmenopause & $12 / 149$ & $15 / 136$ & 0.333 & I & 0.564 \\
\hline Diabetes (yes) & 33 & 31 & 0.222 & I & 0.882 \\
\hline pre-/postmenopause & $7 / 26$ & $8 / 23$ & 0.019 & I & 0.890 \\
\hline Cardiovascular comorbidities (yes) & 148 & 136 & 0.964 & I & 0.326 \\
\hline pre-/postmenopause & $18 / 130$ & $24 / 112$ & 1.285 & I & 0.257 \\
\hline Thyroid pathologies (yes) & 28 & 35 & 0.222 & I & 0.637 \\
\hline pre-/postmenopause & $1 \mathrm{I} / \mathrm{I7}$ & $10 / 25$ & 0.394 & 1 & 0.530 \\
\hline Obesity (yes) & 9 & 16 & 1.486 & I & 0.223 \\
\hline pre-/postmenopause & $6 / 3$ & $7 / 9$ & 0.468 & I & 0.494 \\
\hline Reproductive system pathologies (yes) & 31 & 39 & 0.773 & I & 0.379 \\
\hline pre-/postmenopause & $15 / 16$ & $30 / 9$ & 4.946 & I & 0.02 \\
\hline Psychiatric diseases (yes) & 77 & 75 & 0.015 & I & 0.903 \\
\hline pre-/postmenopause & $30 / 47$ & $25 / 50$ & 0.306 & I & 0.580 \\
\hline Other diseases (yes) & 72 & 145 & 38.981 & I & $0.000 \mathrm{I}$ \\
\hline pre-/postmenopause & $13 / 59$ & $38 / 107$ & 1.354 & I & 0.245 \\
\hline Drug intake (yes) & 156 & 210 & 22.457 & I & 0.0001 \\
\hline pre-/postmenopause & $41 / 113$ & $42 / 155$ & 1.069 & I & 0.301 \\
\hline
\end{tabular}

Abbreviations: AD, Alzheimer's disease; df, degrees of freedom. 
and the presence of other pathologies $(P<0.01)$ were significantly higher in the control patients, thereby confirming their role in AD.

\section{Discussion}

The results of our study show that female controls went through menopause earlier and had a greater incidence of surgical menopause and reproductive-system pathologies before menopause than did women with $\mathrm{AD}$; also, the reproductive life span was shorter. Moreover, the control patients were more likely to have taken HRT than were the AD patients. These findings are strictly related to each other; in particular, relationships were found between earlier menopause and HRT intake and between reproductive system pathologies occurring before menopause and surgical menopause. No significant differences were found between the $\mathrm{AD}$ patients and controls with respect to other reproductive life milestones, ie, age at puberty, previous abortions, number of pregnancies, and contraceptive therapy.

Our results suggest that among reproductive life events, only menopause and HRT intake distinguished the AD patients from the controls. These findings are in agreement with recent data showing a possible protective role for HRT. Some authors ${ }^{5,6,8-10}$ have hypothesized that a critical period around menopause exists during which the prescription of HRT might reduce the risk of developing AD at an older age. In our sample, a higher percentage of controls went through menopause before 45 years of age, as compared to AD patients; we can hypothesize that the control patients began HRT at an earlier age, with a possible protective effect. A higher risk of developing cognitive deficits following surgical menopause has been reported; ${ }^{13,17}$ conversely, we observed a higher incidence of surgical menopause in our control patients, but it is possible that their higher HRT intake protected these subjects from the dramatic estrogen loss that occurs following surgical menopause.

The control women also had a greater incidence of other pathologies (not known to be potential risk factors for dementia) and a higher drug intake than did the AD patients. In the multivariate analysis, only the presence of other pathologies was related to the lower risk of developing dementia, while no significant differences were found for drug intake, regardless of the type of drug. There was a relationship between drug intake and the presence of other pathologies (in fact, people with more pathologies generally take more drugs); however, it is difficult to explain how their relationship relates to the risk of developing AD. These results may be related to the type of enrollment (as explained below), with the control patients having greater recall than those with cognitive disease. It is noteworthy that we did not find any differences between the AD patients and the controls with respect to cardiovascular comorbidities or hypertension, which are factors capable of contributing to cognitive decline.

The multivariate analysis also showed that a higher level of education is related to a lower risk of developing AD. This relationship, irrespective of gender, has been clearly demonstrated in the literature. ${ }^{27,28}$ Education appears to have a protective effect on the development of $\mathrm{AD}$; in addition to mentally and socially integrated lifestyles, education may postpone the onset of clinical dementia and therefore reduce its incidence. ${ }^{28}$ These data have been explained by the concept of a "cognitive reserve:" patients with a higher level of education have a greater cognitive reserve. ${ }^{28}$ Undoubtedly, an important limitation of our study is the type of enrollment. We designed this study to explore the reproductive life events preceding the onset of dementia, and the case-controlled approach was deemed useful for determining the relevance of these factors. We chose to enroll controls from among neurological outpatients to increase female participation and obtain a more selected population in terms of cognitive status. Another limitation may be the multicenter design, which nonetheless ensures control over geographical and ethnic variables. The controls' mean age was lower for the Pavia clinic; however, the multivariate analysis excluded any predictive role for this factor.

Although we are aware that our results cannot be generalized to the entire population, nonetheless, to our knowledge, this is the first study in which all of the reproductive life events, not only menopause, were evaluated as potential factors capable of influencing the development of AD.

$\mathrm{AD}$ is a very complex, multifactorial disease. Besides genetic predisposition, which plays a major role, gender is an important factor in influencing disease susceptibility as well as disease evolution and outcomes..$^{2,3,29,30}$ In women, menopause and HRT can differentially modulate the clinical manifestations of the disease, but they do not seem to have a predictive role in its development.

\section{Disclosure}

The authors report no conflicts of interest in this work.

\section{References}

1. Genazzani AR, Pluchino M, Luisi S, Luisi M. Estrogen, cognition and female ageing. Hum Reprod Update. 2007;13:175-187.

2. Musicco M. Gender differences in the occurrence of Alzheimer's disease. Funct Neurol. 2009;24:89-92. 
3. Gambassi G, Lapane KL, Landi F, Sgadari A, Mor V, Bernabie R; the Systematic Assessment of Geriatric drug use via Epidemiology (SAGE) Study Group. Gender differences in the relation between comorbidity and mortality of patients with Alzheimer's disease. Neurology. 1999; 53:508-516.

4. Azad NA, Al Bugami M, Loy-English I. Gender differences in dementia risk factors. Gend Med. 2007;4:120-129.

5. Czlonkowska A, Ciesielska A, Gromadzka G, Kurkowska-Jastrzebska I. Gender differences in neurological diseases: role of estrogens and cytokines. Endocrine. 2006;29:243-256.

6. Ancelin ML, Ritchie K. Lifelong endocrine fluctuations and related cognitive disorders. Curr Pharm Des. 2005;11:4229-4252.

7. Craig MC, Murphy DGM. Estrogen therapy and Alzheimer's dementia. Ann NY Acad Sci. 2010;1205:245-253.

8. Rocca WA, Bower JH, Maraganore DM, et al. Increased risk of cognitive impairment or dementia in women who underwent oophorectomy before menopause. Neurology. 2007;69:1074-1083.

9. Sherwin BB. Estrogen and cognitive functioning in women. Endocr Rev. 2003;24:133-151.

10. Greendale GA, Huang MH, Wight RG, et al. Effects of the menopause transition and hormone use on cognitive performance in midlife women. Neurology. 2009;72:1850-1857.

11. Hogervorst E, Bandelow S. Sex steroids to maintain cognitive functions in women after menopause: a meta-analyses of treatment trials. Maturitas. 2010;66:56-71.

12. Henderson W. Action of estrogens in the aging brain: dementia and cognitive aging. Biochim Biophys Acta. 2010;10:1077-1083.

13. Henderson VW, Sherwin BB. Surgical versus natural menopause: cognitive issues. Menopause. 2007;14:572-579.

14. Kok HS, Kuh D, Cooper R, et al. Cognitive function across the life span course and the menopausal transition in a British birth cohort. Menopause. 2006;13:4-5.

15. Weber M, Mapstone M. Memory complaints and memory performances in the menopausal transition. Menopause. 2010;16:694-700.

16. Soares CN, Maki PM. Menopausal transition, mood, and cognition: an integrated view to close the gaps. Menopause. 2010;17:812-814.

17. Nappi RE, Sinforiani E, Mauri M, Bono G, Polatti F, Nappi G. Memory functioning at menopause: impact of age in ovariectomized women. Gynecologic and Obstet Invest. 1999;47:29-36.
18. Bonomo SM, Rigamonti AE, Giunta M, et al. Menopausal transition: a possible risk factor for brain pathologic events. Neurobiol Aging. 2009;30:71-80.

19. Debette S, Seshadri S, Beiser A, et al. Midlife vascular risk factor exposure accelerates structural brain aging and cognitive decline. Neurology. 2011;77:461-468.

20. Mckhann G, Drachman D, Folstein M, Katzman R, Price D, Stadlan EM. Clinical diagnosis of Alzheimer's disease: report of the NINCDS-ADRDA Work Group under the auspices of Department of Health and Human Services Task Force on Alzheimer's Disease. Neurology. 1984;34:939-944.

21. Folstein MF, Folstein SE, McHugh PR. "Mini-mental state.” A practical method for grading the cognitive status of patients for the clinician. J Psychiatr Res. 1975;12:189-198.

22. Measso G, Cavarzeran F, Zappalà G, et al. The Mini-Mental State Examination: normative study of an Italian random sample. Develop Neuropsychol. 1993;9:77-85.

23. Lawton MP. Scales to measure competence in everyday activities. Psychopharmacol Bull. 1988;24:609-614.

24. Lawton MP, Brody EM. Assessment of older people: self-maintaining and instrumental activities of daily living. Gerontologist. 1969;9: 179-186.

25. Cummings JL, Mega M, Gray K, Rosenberg-Thompson S, Carusi DA, Gornbein J. The Neuropsychiatric Inventory: comprehensive assessment of psychopathology in dementia. Neurology. 1994;44:2308-2314.

26. Hughes CP, Berg L, Danziger WL, Coben LA, Martin RL. A new clinical scale for the staging of dementia. Br J Psychiatr. 1982;140:566-572.

27. Scarmeas N, Albert SM, Manly JJ, Stern Y. Education and rates of cognitive decline in incident Alzheimer's disease. J Neurol Neurosurg Psychiatry. 2006;77:308-316.

28. Fratiglioni L, Wang HX. Brain reserve hypothesis in dementia. J Alzheimers Dis. 2007;12:11-22.

29. Noale M, Maggi S, Minicuci N, et al; ILSA Working Group. Dementia and disability: impact on mortality. The Italian Longitudinal Study on Aging. Dement Geriatr Cogn Disord. 2006;16:7-14.

30. Sinforiani E, Citterio A, Zucchella C, et al. Impact of gender differences on the outcome of Alzheimer's disease. Dement Geriatr Cogn Disord. 2010;30:147-154.
Neuropsychiatric Disease and Treatment

\section{Publish your work in this journal}

Neuropsychiatric Disease and Treatment is an international, peerreviewed journal of clinical therapeutics and pharmacology focusing on concise rapid reporting of clinical or pre-clinical studies on a range of neuropsychiatric and neurological disorders. This journal is indexed on PubMed Central, the 'PsycINFO' database and CAS.

\section{Dovepress}

The manuscript management system is completely online and includes a very quick and fair peer-review system, which is all easy to use. Visit http://www.dovepress.com/testimonials.php to read real quotes from published authors. 